\title{
¿Cuál es el futuro de la medicina basada en la evidencia?
} MEDLINE/PubMed, Tripdatabase, Cochrane library, etc., facilitan el acceso a la información disponible a través de Internet. Para poder utilizar la evidencia de la investigación se necesita tiempo, esfuerzo y los conocimientos necesarios para conseguir información adecuada entre el gran volumen de investigación que se publica. Es necesario desarrollar estrategias claras y eficaces para filtrar, discriminar, digerir y actuar sobre la nueva investigación y obtener ventajas para los pacientes. Para ello se debe fomentar el desarrollo de dos principales líneas de actuación: por una parte se deben adquirir conocimientos para conseguir la evidencia y, por otra, se debe llevar la evidencia a la práctica clínica (1).

Por desgracia no siempre se dispone de revisiones sistemáticas, metaanálisis, ensayos clínicos, etc., con una buena metodología y una serie de casos suficientes en los que basar la decisión adecuada sin posibilidad de equivocación. A ello hay que añadir que, en ocasiones, los estudios consultados aportan resultados contradictorios sobre la información que se precisa. Se debe decidir cuál es la verdadera y más adecuada en cada momento y para cada paciente, pudiendo llegar a convertirse en un problema de difícil solución. Esto se puede agravar si no se dispone de una metodología que permita, de forma objetiva, realizar una evaluación crítica y discriminativa de la información recibida. Esta decisión es de suma importancia cuando se pretende modificar la práctica clínica a partir de la mejor información disponible (1).

Para dar respuesta a esta problemática surgió la Medicina Basada en la Evidencia (MBE) o Asistencia Sanitaria Basada en la Evidencia (ASBE), que desde sus inicios contaba y cuenta con numerosos detractores. La MBE integra la mejor evidencia científica disponible con la experiencia y la habilidad clínica, con el conocimiento de la fisiopatología y con las preferencias de los pacientes, para la adecuada toma de decisiones pertinentes a la atención de alta calidad (2).

El objetivo final que busca la ASBE es la adecuación de la práctica médica a la investigación clínica disponible de modo que, una vez localizada y evaluada, sea aplicada para mejorar la propia práctica clínica en beneficio de los pacientes (2).

Parecería una contradicción abogar por la superioridad de un nuevo tratamiento o técnica cuando no existen demostraciones claras al respecto. La ASBE no rechaza las innovaciones cuando no existen pruebas suficientes, sino que cuando no existen evidencias definitivas se deben aceptar las disponibles. No se debe olvidar que los médicos que se mantienen actualizados lo hacen en función de su capacidad de valorar críticamente la literatura y de distinguir entre las pruebas que aportan una evidencia fuerte o más débil. De esta forma, se puede seleccionar y recomendar las pruebas diagnósticas y las estrategias terapéuticas más eficiente en cada caso (2).

En la actualidad unos deseos se han convertido en unas exigencias y una necesidad de proporcionar a los clínicos y a los pacientes la oportunidad de conseguir la atención más eficaz en función de las evidencias procedentes de una investigación de máxima calidad. 
La MBE no es una medicina para reducir costes. Cuando se dirige hacia el beneficio del paciente en particular la MBE identifica y aplica las intervenciones más eficaces para maximizar la calidad y cantidad de vida de los pacientes, y esto puede suponer un aumento de los costes de su asistencia más que una disminución de los mismos.

Practicar MBE consiste en localizar las mejores evidencias externas con las que responder a nuestras preguntas clínicas; en el caso de las pruebas diagnósticas, en localizar estudios transversales adecuados realizados en pacientes sospechosos de padecer la patología de estudio; en los pronósticos, seleccionar estudios de seguimiento establecidos en un punto precoz y uniforme del curso clínico de la enfermedad.

La asistencia a los usuarios crea continuamente la necesidad de información, que se debe convertir en preguntas susceptibles de respuesta. La mejor evidencia se puede localizar en la historia clínica, la exploración física, diagnósticos de laboratorio y publicaciones (MEDLINE, Embase, Cochrane Library, PubMed, Bandolier, etc.). Se debe evaluar la evidencia en términos de validez (aproximación a lo correcto) y utilidad (aplicabilidad clínica). Es fundamental la aplicación de los resultados de la evaluación crítica a la práctica clínica para, finalmente, evaluar nuestro rendimiento.

Cuando planteamos la selección de un tratamiento se deben intentar evitar enfoques no experimentales que llevan a conclusiones falsamente positivas sobre la eficacia. Los ensayos randomizados y las revisiones sistemáticas son esenciales para juzgar si un tratamiento produce más beneficio que daño. Sin embargo, ciertas preguntas sobre tratamientos no requieren ensayos randomizados, o no se puede esperar a que se lleven a cabo.

Una vez que el clínico identifica la mejor evidencia científica externa disponible debe valorar si es pertinente su aplicación eficiente al paciente. Para ello debe tener en cuenta las preferencias y condiciones de cada paciente. Finalmente, el médico debe decidir cómo integrar las evidencias externas en una decisión clínica determinada. Esto supone entender la magnitud del impacto de un tratamiento o la utilidad relativa de diversas pruebas diagnósticas.

Para intentar solventar esta problemática se inició la elaboración de guías de práctica clínica (GPC), llegando a producirse un enorme número de guías, por lo que vuelve a presentarse el mismo dilema aunque a menor escala. ¿Qué guía es la mejor y, por lo tanto, la que se debe utilizar? Paulatinamente se ha ido incorporando la MBE a la elaboración de las diferentes GPC, en un intento de mejorar la calidad de las mismas. El desarrollo de herramientas como AGREE permite hacer una evaluación sistemática por apartados, lo que ayuda al clínico a elegir la GPC más adecuada para cada decisión clínica $(1,3)$.

Los niveles de evidencia, la calidad y el grado de recomendación constituyen la base de las GPC basadas en la evidencia, permitiendo al clínico valorar la investigación publicada, así como determinar su validez y aplicabilidad en la práctica clínica. Diferentes centros de elaboración de GPC, instituciones y sociedades científicas han desarrollado distintos sistemas de clasificación para evaluar y estructurar la evidencia y establecer los grados de recomendación. En la mayoría de las clasificaciones se opta por señalar unos niveles de evidencia y grados de recomendación que solo tienen en cuenta los estudios sobre intervenciones terapéuticas. Actualmente hay más de 100 sistemas de clasificación para valorar la calidad de la evidencia lo que, en ocasiones, provoca cierto grado de confusión cuando se consultan diferentes GPC (2-4).

Aguilera y cols., en su artículo "Niveles de evidencia y grados de recomendación en kinesiología; una comparación entre 5 sistemas de clasificación; estudio piloto", plantean esta problemática y se preguntan si la herramienta de clasificación GRADE podría facilitar la comprensión de los niveles de evidencia y grados de recomendación con respecto a las clasificaciones de NIC, SIGN, CEBM y CTPHL a la hora de tomar una decisión clínica para los kinesiólogos que trabajan en el área de la Rehabilitación traumatológica (5). En este artículo Aguilera y cols. consideran que el sistema de clasificación GRADE es la forma más simple para que los kinesiólogos puedan entender la evidencia científica, considerando las limitaciones que se enumeran en el estudio.

La utilización de niveles de evidencia comunes en las diferentes GPC y estudios de investigación, y el empleo de herramientas como GRADE, de fácil aplicación y comprensión, serán aspectos que ayudarán y facilitarán la práctica integral de la MBE, que será posible cuando mejore 
la tecnología para manejar e integrar la literatura científica y exista una mayor receptividad a la metodología de la MBE.

No obstante, se debe ser crítico con la MBE y no promover indiscriminadamente su práctica hasta que no se disponga de mejores evidencias sobre su capacidad para mejorar los resultados en los pacientes. Por desgracia, la MBE aporta poca información sobre los efectos adversos; las revisiones sistemáticas, en ocasiones, conducen a conclusiones de forma apresurada y algunos clínicos adoptan cambios con demasiada rapidez. Con frecuencia se presentan dudas y preguntas que continuamente tratamos de responder:

- ¿Estamos haciendo las cosas correctamente?

- ¿Cuál es la evidencia de la evidencia?

- ¿Por dónde debemos seguir? ¿Cuál es el camino?

Con el transcurso del tiempo los éxitos y fracasos, así como la integración de los resultados obtenidos con la práctica de la MBE, nos indicarán cuál es el mejor camino a seguir y cuál debemos abandonar.

\section{F. Neira-Reina y J.L. Ortega-García}

Servicio de Anestesiología, Reanimación y Tratamiento del Dolor. Hospital Universitario Puerto Real. Cádiz.

\section{BIBLIOGRAFÍA}

1. Neira F, Ortega JL, Martínez J, Gálvez R, De la Torre R, Torres LM. Evaluación mediante AGREE de guías de práctica clínica en dolor postoperatorio. Rev Esp Anestesiol Reanim 2005;52:75-80.

2. Neira F, Ortega JL, Torres LM. Tratamiento farmacológico del dolor. Recomendaciones de las guías de práctica clínica. Madrid: EdikaMed; 2008.

3. García Gutiérrez JF. Métodos y estrategias para la implementación de las guías de práctica clínica (GPC). Guías de Práctica Clínica. AETSA, Universidad de Málaga; 2002.

4. Ebell MH, Siwek J, Weiss BD, Woolf SH, Susman J, Ewigman B, et al. Strength of Recommendation Taxonomy (SORT): A patient-centered approach to grading evidence in the medical literature. Am Fam Physician 2004;69:548-56.

5. Harbour R, Miller J. A new system for grading recommendations in evidence based guidelines. BMJ 2001;323:334-6. 